\title{
Reappraisal on the Management of Primary Dysmenorrhoea in Adolescents
}

\author{
Najimudeen $\mathrm{M}^{*}$, Myo Hla Myint, Masharudin A. W \\ International Medical School (IMS), Management and Science University, Malaysia
}

DOI: $10.36348 /$ sijog.2020.v03i09.001

| Received: 21.08.2020 | Accepted: 29.08.2020 | Published: 03.09.2020

*Corresponding author: Mohamed Najimudeen

Abstract

Primary dysmenorrhea is painful menstrual period without any underlying pathological cause. It is a common problem among adolescents. Despite the high prevalence, primary dysmenorrhea is often poorly treated, disregarded by health professionals, pain researchers, and the adolescents themselves. They may accept it as a normal part of the menstrual cycle. Increased levels of prostaglandins E2 and F2 $\alpha$ cause dysrhythmic myometrial contraction, hypercontractility, increased muscle tone leads to ischaemia and pain. It affects the quality of life and performance. Non-steroidal antiinflammatory drugs (NSAIDs) are the first line of treatment. Progesterone significantly relieves the pain in subsequent cycles. Combined oral contraceptives (COC) are invaluable in the management of dysmenorrhoea.

Keywords: Primary dysmenorrhoea, adolescents, Prostaglandins, NSAIDs, Progesterone, COC.

Copyright @ 2020: This is an open-access article distributed under the terms of the Creative Commons Attribution license which permits unrestricted use, distribution, and reproduction in any medium for non-commercial use (NonCommercial, or CC-BY-NC) provided the original author and source are credited.

\section{INTRODUCTION}

Dysmenorrhoea is painful menstrual period. Dysmenorrhea occurs throughout the adolescent, sexual maturation, and climacteric periods until menopause [1]. The dysmenorrhoea is divided into primary and secondary. The primary dysmenorrhoea is without any underlying organic cause. The secondary dysmenorrhea is associated with organic diseases such as endometriosis, adenomyosis, pelvic inflammatory disease and uterine myoma. The effective treatment for both primary and secondary dysmenorrhea includes NSAIDs that inhibit PG production, progesterone only tablets, combined low-dose oestrogen/progestin (EP) tablets and levonorgestrel-releasing intrauterine system that suppress ovulation and/or endometrial proliferation to suppress PG production. In secondary dysmenorrhoea the organic diseases should be preferentially treated [2].

Primary dysmenorrhea is spasmodic cramping in the lower abdomen, just before or during menstruation in the absence of any identifiable macroscopic pelvic pathology. Primary dysmenorrhea occurs only during ovulatory cycles [3].
The secondary dysmenorrhoea is due to identifiable pelvic pathology. It occurs with ovulatory and anovulatory cycle [4].

\section{MATERIALS AND METHODS} dysmenorrhoea.

This is a review study of literature on primary

\section{DISCUSSION \\ Prevalence}

The prevalence of primary dysmenorrhea is highly underestimated. Difficult to determine since affected teenagers seldom seek medical treatment. Most adolescents do not seek medical attention for their pain and most cases go undocumented because many teenagers still believe that menstrual pain is a normal part of the female menstrual cycle [5].

In a cross-sectional study with 1092 girls from 15 public secondary schools and 3 ethnic groups in the Federal Territory of Kuala Lumpur Malaysia, $74.5 \%$ of the girls who had reached menarche had dysmenorrhea. $51.7 \%$ of these girls reported that it affected their concentration in class. $50.2 \%$ restricted their social activities. $21.5 \%$ missed school and $12.0 \%$ had poor school performance. They had poor concentration, absenteeism, and restriction of social and recreational 
activities attributed to dysmenorrhea. Only $12.0 \%$ had consulted a physician, and $53.3 \%$ did nothing about their conditions [6].

Very severe primary dysmenorrhea estimated to affect $10-25 \%$ of women of reproductive age. Dysmenorrhea appears to be the most common gynaecological disorder in women irrespective of nationality and age. Dysmenorrhea is a common symptom in teenagers and affects up to $40-50 \%$ of young women, with severe forms in $15 \%$ [7].

Abere et al., studied menstrual problems in 459 female students. The prevalence of primary dysmenorrhea among female students was found to be $64.7 \%$ (95\% CI; 60.2-69.2\%). Around 61\% reported moderate intensity of menstrual pain and $50.7 \%$ complain about lower abdominal pain. Sixty-five percent of study participants reported that absenteeism from school was the impact of menstrual pain. Having irregular monthly menstrual cycle $(\mathrm{AOR}=1.70,95 \%$ $\mathrm{CI} ; 1.02,2.84)$ and positive family history of dysmenorrhea $(\mathrm{AOR}=5.19,95 \% \mathrm{CI}: 3.21,8.37)$ were significantly associated with primary dysmenorrhea [8].

The prevalence of dysmenorrhea was studied in a random sample of 19-year-old women from an urban Swedish population. Dysmenorrhea was reported by $72 \%$ of the women. Fifteen percent suffered from dysmenorrhea which limited daily activity and was unimproved by analgesics. Dysmenorrhea occurred significantly ( $\mathrm{p}$ less than 0.01 ) more often in women not using oral contraceptives. A significant correlation ( $p$ less than 0.01) was found between early menarche and an increased severity of dysmenorrhea. There was a significant correlation ( $\mathrm{p}$ less than 0.01 ) between the severity of dysmenorrhea and the amount of menstrual flow. Smokers as compared to non smokers had significantly (p less than 0.01) less dysmenorrhea [9].

Different aspects of the menstrual pattern including dysmenorrhea, premenstrual tension and working performance during menstruation was studied in three different investigations. The first study includes 5458 adolescent girls aged 10 to 20 . The incidence of invariably painful menstruation was 7.2 per cent during the first gynaecological year and 26 per cent five years later. A correlation was found between mothers and daughters for dysmenorrhea as well as for premenstrual tension. In a second study including 331 girls aged 13 to 20 years the overall absence from school due to pain was 23.4 per cent. The frequency of dysmenorrhea increased from 36 per cent to 56.5 per cent between 13 14 years and 17-20 years. In the third study sociomedical aspects of menstrual pattern were studied in 865 adult women. Approximately 88 per cent had premenstrual tension and almost 45 per cent had menstrual pain [10].
Risk factors for dysmenorrhea, but not specifically primary dysmenorrhea, include smoking, earlier age at menarche, longer and heavier menstrual flow, higher BMI, alcohol consumption, family history of dysmenorrhea [11]. Dysmenorrhea is increased with smoking [12].

\section{Pathophysiology}

There are nine classes of PGs: PGA through PGI; within which individual PG are denoted by numerical subscripts. The two types of PGs that are implicated in the pathogenesis of pain in primary dysmenorrhoea are $\mathrm{PGF}_{2 \alpha}$ and $\mathrm{PGE}_{2}$; however, $\mathrm{PGF}_{2 \alpha}$ appears to be of particular importance. While $\mathrm{PGE}_{2}$ may result in either myometrial contraction or relaxation, $\mathrm{PGF}_{2 \alpha}$ always causes potent vasoconstriction of uterine blood vessels, and myometrial contractions. There also is evidence that $\mathrm{PGF}_{2 \alpha}$ lowers the threshold for pain perception by sensitizing nerve endings to pain .Studies investigating uterine blood flow using Doppler ultrasonography have shown that the strong and abnormal uterine contractions in women with dysmenorrhea during menstruation are associated with reduced uterine blood flow and resultant myometrial ischemia, and hence pain .Thus, during menstruation, excessive release of PGs by the endometrium results in hypercontractility of the uterus, and subsequent uterine muscle ischemia and hypoxia. The contraction of the ischemic uterus therefore is the likely cause of dysmenorrhoeic pain [13].

Primary dysmenorrhoea is caused by myometrial contractions producing uterine ischaemia in response to elevated levels of prostaglandins E2 and F2 $\alpha$ released from shedding secretory endometrium. Myometrial contractions can last several minutes, with uterine pressures of more than $60 \mathrm{~mm}$ of mercury, resulting in severe pain. Prostaglandins may also cause commonly associated symptoms, including nausea, vomiting, dizziness and faintness, and these also tend to recur from one cycle to the next. The pathophysiology is related to prostaglandins and leukotrienes, both mediators of inflammation. One study that measured prostaglandin $\mathrm{F}_{2 \mathrm{a}}\left(\mathrm{PGF}_{2 \alpha}\right)$ activity in menstrual fluid from tampons found that dysmenorrhoeic women had twice the $\mathrm{PGF}_{2 \alpha}$ activity level as eumenorrheic women. In addition, higher urinary leukotrienes levels have been found in adolescent girls with dysmenorrhea, adding support for the idea that these inflammatory mediators play a role in dysmenorrhea [14].

In normal women, the uterus has well defined contraction patterns that are influenced by sex steroids, prostaglandins, and other uterotonic substances throughout the menstrual cycle. . During menstruation in normal women, the uterine basal tone is minimal (less than $10 \mathrm{~mm} \mathrm{Hg}$ ). In patients with primary dysmenorrhea, the basal tone is elevated more than 10 $\mathrm{mmHg}$. This may increase to $120 \mathrm{~mm} \mathrm{Hg}$ and in severe dysmenorrhoea $150 \mathrm{~mm} \mathrm{Hg}$. There is an increase in the 
number of contractions per 10 minutes (more than 4 or 5), and nonrhythmic or incoordinate uterine contractions are noted the cyclic activity of the nonpregnant human uterus: a new method for recording intrauterine pressure. These abnormalities lead to poor uterine perfusion and oxygenation, thus giving rise to pain. In most but not all women with primary dysmenorrhea, there is increased endometrial secretion of menstrual prostaglandin F2 (PGF2) during the menstrual phase. The intensity of the menstrual cramps and associated symptoms of dysmenorrhea are directly proportional to the amount of PGF2 released [15].

\section{Presenting Features}

The primary dysmenorrhea usually occurs in adolescence. The pain usually has a clear and predictable temporal pattern, beginning just before or with the onset of menstruation. The pain typically lasts for $8-72$ hours. It is most severe during the first or second day of menstruation. It may radiate to the back and thighs. In addition, systemic symptoms such as nausea, vomiting, diarrhoea, fatigue and insomnia frequently accompany the pain [16]. Young girls can experience dysmenorrhoea few months or years after the menarche. After the menarche it may take 1-2 years for the maturity of hypothalamo- pituitary ovarian (HPO) axis.

Poor sleep quality including disturbances in sleep onset had been reported with severe dysmenorrhea. As a result of these related symptoms, dysmenorrhea is the leading cause of recurrent shortterm school absenteeism for adolescent girls In one study, $12 \%$ of adolescent girls and young women aged 14-20 years lost days of school or work each month because of dysmenorrhea. An association between premenstrual syndrome and dysmenorrhea in adolescent girls also has been demonstrated [17, 18].

A study of Japanese high school students showed that the rates of prevalence of premenstrual syndrome and premenstrual dysphoric disorder increased with the severity of dysmenorrhea. There is an increased risk of depression and anxiety in adolescents with dysmenorrhea .Health-related qualityof-life questionnaires administered to adolescents with dysmenorrhea have demonstrated negative effect on physical and psycho-social functioning [19].

Primary dysmenorrhea, which is often found in young women, may be an important sign of future endometriosis. A report has shown that a history of primary dysmenorrhea increases the risk of endometriosis [20].

\section{Diagnosis \\ History}

History is the most important part in the diagnosis of primary dysmenorrhoea Spasmodic menstrual cramps, that begin only a few hours before or with the onset of menstrual flow, the symptoms of primary dysmenorrhea lasts only 2-3 days. The pains are most intense on the first or second day of the menstrual flow, or more precisely the first 24-36 hours, consistent with the time of maximal prostaglandin release into the menstrual fluid (vide infra). The cramps are frequently accompanied by backache, nausea, vomiting, and diarrhoea [21].

Menstrual history: The onset of menarche, regularity of the menstrual cycle, duration of menstrual period and the amount of bleeding (flooding, passing clots, night changes) should be obtained.

The history of dysmenorrhoea should be noted in detail. On what day and time of the pain begins in relation to the menstrual period, the intensity and progression of the period should be noted.

Presence of systemic symptoms such as nausea, vomiting, anxiety, depression and insomnia to be recorded.

Sexual history to be obtained. The undiagnosed pelvic inflammatory disease can be causing secondary dysmenorrhoea.

\section{Examination}

There will be no positive findings in primary dysmenorrhoea. The typical history of pain will suggest primary dysmenorrhoea. Vaginal and rectal examinations are not necessary.

If the secondary dysmenorrhoea is suspected, examination of external genitalia, vaginal vault, cervix and bimanual examination are indicated.

\section{Investigations}

In primary dysmenorrhoea, investigations are also inconclusive. However if the secondary dysmenorrhoea is suspected, full blood count, Sexually transmitted disease (STD) profile, CA125, urine microscopy, ultrasound examination, Hysterosalpingogram maybe indicated.

The differential diagnoses include ovarian cysts and non-gynaecological conditions, such as inflammatory bowel disease, irritable bowel syndrome, constipation and painful bladder syndrome; although a cyclical pattern of pain is most typical of primary dysmenorrhoea.

\section{Management \\ Non-Steroidal Anti-Inflammatory Drugs (NSAIDs) \\ NSAID is the first line of treatment of primary dysmenorrhoea [22].}

NSAID relieve primary dysmenorrhea by inhibiting endometrial prostaglandin production but also have direct analgesic properties at the central 
nervous system level. Nonsteroidal anti-inflammatory drugs do not appear to affect the development of the endometrium when given during the menstrual phase for primary dysmenorrhea but directly inhibit COX activity and therefore suppress endometrial prostaglandin biosynthesis and release [23].

NSAIDs act by inhibiting the enzyme that catalyses the conversion of arachidonic acid to cyclic endoperoxides, namely cyclo-oxygenase (COX) This in turn inhibits the production of PGs. Suppression of PG formation results in a reduction in uterine PG secretion and thus less vigorous uterine contractions, many NSAIDs provide effective relief from dysmenorrhoea. Thus, NSAIDs alleviate pain predominantly through the suppression of endometrial PG synthesis

NSAIDs such as mefenamic acid, should be commenced 2-3 days prior to withdrawal bleeds, as they can act to lower circulating prostaglandin levels and also reduce menstrual flow by up to 30 per cent [24].

The majority of patients with dysmenorrhoea will respond to NSAIDs if these are taken correctly. It is essential that all women taking NSAIDs for primary dysmenorrhoea fully understand this concept if they are to benefit appropriately from their use. Several studies have shown that patients reporting they had found NSAIDs ineffective had, in fact, not taken them until many hours after the pain had begun [25].

These drugs are prostaglandin synthetase inhibitors, which act by inhibiting the cyclooxygenase enzymes (COX enzymes) hence preventing the formation of prostaglandins. Therefore these medications cannot substantially reverse pain already present. With the onset of severe pain the patients take these tablets. The results are poor and the patients are frustrated. It is a common mistake that we do not tell the patient that these medications should be taken one or two days before the onset of the menstrual period. Generally these teenagers have some premenstrual symptoms such as breast discomfort, bloated feeling and headache one or two days before the period. They should take the medication at that point- one or two days before the onset of menstrual period [26].

There are more than 70 well-conducted randomised controlled trials (RCTs) testifying to the effectiveness of NSAIDs in the prevention of primary dysmenorrhoea when compared to placebo and other treatments. Studies have also shown that no particular NSAID stands out as significantly more effective than any other. Naproxen, mefenamic acid and ibuprofen are those most commonly and effectively used in Australian practice at the present time and can be bought over the counter. One limiting factor is side effects, mostly gastro-intestinal; patients should be advised to take preparations with food or milk and to take them only as long as needed each cycle. Most young women quickly become aware of the systemic and pelvic symptoms of an incipient period, and will be helped by keeping a menstrual calendar, so that the chosen NSAID can then be taken at an appropriate time prior to the onset of pain.

It is also useful to make practical suggestions to this younger age group and recommending that a couple of tablets are kept at hand in the school bag together with an emergency supply of sanitary products is often helpful. Many young girls either before their first period or in the first few years following menarche live in terror of leaving 'little red puddles' on clothing and so forth and are greatly reassured by such strategies.

A Cochrane review of 73 randomized controlled trials (RCTs) demonstrated strong evidence to support that NSAIDs are the first-line treatment for primary dysmenorrhea. The choice of NSAID should be based on effectiveness and tolerability for the individual patient, because no NSAID has been proven more effective than others. Medications should be taken one to two days before the anticipated onset of menstrual period, and continued on a fixed schedule for two to three days [27].

Adverse effects of NSAIDs include gastrointestinal symptoms, central nervous system symptoms, nephrotoxic and hepatotoxic effects, hematologic abnormalities, bronchospasm, fluid retention, and oedema,. However with a 3-day regimen used in primary dysmenorrhoea who are usually young and healthy, adverse effects are infrequent, well tolerated, and usually confined to gastrointestinal symptoms such as nausea, indigestion, heart burn, and vomiting.

\section{Progesterone Only Preparations}

Prostaglandin production is controlled by progesterone. Just prior to menstruation, progesterone levels drops and prostaglandin levels increase Luteal phase is crucial for the increased production of progesterone. Therefore dysmenorrhea occurs only with ovulatory cycles. This could explain why primary dysmenorrhea onset is shortly after menarche and why dysmenorrhea responds well to ovulatory inhibition [28].

Hormonal progestins-only treatment produces a benefit on menstrual pain, causing endometrial atrophy and inhibiting ovulation. Several long-acting reversible progestin contraceptives have been found to be effective treatments for primary dysmenorrhea. These include 52-mg (20 $\mu \mathrm{g} /$ day $)$ levonorgestrelreleasing intaruterine system, the etonogestrel-releasing subdermal implant, and depot medroxyprogesterone [29]. 
Dienogest (DNG) is a progestin derived from 19-nortestosterone but is different in structure from other progestins in this class. The $17 \alpha$-ethinyl group, typical of many 19-nortestoerone progestin derivatives, is replaced by a $17 \alpha$-cyanomethyl group in DNG [5]. Similar to the other agents in its class, DNG exerts a strong pregestational effect on the endometrium. However, unlike other 19-nortestosterone derivatives, it is characterized by an overall antiandrogenic hormonal milieu.

Dienogest reduces endometriotic lesions through a number of biological mechanisms. Dienogest is associated with relatively moderate inhibition of gonadotropin secretion, leading to a modest reduction in the endogenous production of estradiol [40]. When given continuously, dienogest induces a hypoestrogenic, local endocrine environment, causing a decidualization of endometrial tissue followed by atrophy of the endometriotic lesions. Animal studies indicate that dienogest may also reduce plasma oestradiol levels directly, through inducing apoptosis of granulosa cells in the ovary [30].

Yutaka Osuga et al., reported a study conducted at 17 institutions in Japan between April 2017 and January 2019 on the use of DNG as treatment of dysmenorrhoea. A total of 147 subjects who received DNG were evaluated. It was found that DNG is safe and effective in the treatment of dysmenorrhoea [31].

The side effects of Dienogest include menstrual irregularities, headache, back pain, breast discomfort, hot flushes, mood changes, acne, nausea, vomiting, abdominal pain and weight gain. Breakthrough bleeding is the common problem. These side effects are seen in first few months of taking and reduces gradually.

This can be overcome by prescribing combined oral contraceptive pill. This combined pill will eliminate intermenstrual spotting hypo oestrogenic features like hot flushes. Combined pill is much cheaper than progesterone alone.

The influence of different oral contraceptives on the prevalence and severity of dysmenorrhea was investigated in a representative sample of 19-year-old women from an urban Swedish population. The prevalence and severity of dysmenorrhea were significantly $(\mathrm{p}<0.01)$ reduced amongst users of progestogen-dominated oral contraceptives compared to a control group of women who used neither oral contraceptives nor an intrauterine device. However, there was no significant difference in the prevalence and severity of dysmenorrhea between users of oral contraceptives with low progestogen activity and the same control group. Thus, the relative progestogen activity of the oral contraceptive used appears to be of importance for the effective treatment of dysmenorrhea [32].

\section{Combined Oral Contraceptives (COC)}

With the addition of non-steroidal analgesia. Cyclical use of the COCP can reduce dysmenorrhoea by a reduction in prostaglandin load through suppression of ovulation and by lessening myometrium contractility through reduced menstrual flow. Continuous use of the COC may be first-line management in the setting of significant dysmenorrhoea. A withdrawal bleed should be scheduled at the end of the first pill packet, followed by continuous use of the active pills. The pill can be taken continuously until breakthrough bleeding occurs, or a withdrawal bleed can be scheduled at the patient's convenience. A four-day hormone pill break is sufficient to allow for a full withdrawal bleed

A Cochrane analysis in 2001 concluded from four randomized controlled trials that combined oral contraceptive pills (OCPs) with medium-dose oestrogen and first-/second-generation progestogens are more effective than placebo for relief of primary dysmenorrhea [33].

OCPs (oestrogen and progestin) reduce menstrual fluid volume and prostaglandins to within, or even below, normal range [34].

Oral contraceptive pills may also lower the elevated plasma vasopressin levels found in dysmenorrhoeic women and lead to attenuation of the excessive uterine activity. Monophasic and triphasic OCPs are equally effective because there is no significant difference in the prevalence rate of dysmenorrhea between users of these preparations [35].

You et al., studied the opinion of 300 mothers about treating with OCP for their daughters suffering from primary dysmenorrhoea. $48 \%$ of the mothers did not have the proper knowledge about OCP. $45 \%$ worried about weight gain. $37 \%$ felt that use of OCP may lead to infertility and cancer. $12 \%$ had felt that OCP use may increase the sexual activity of their daughters [36].

\section{The Levonorgestrel-Releasing Intrauterine System (LNG-IUS)}

LNG-IUS can be offered for management of adolescent dysmenorrhoea when oestrogen is contraindicated or poorly tolerated. An ultrasound scan to measure the length of the endometrial cavity is not required as a standard-sized LNG-IUS can be safely used in any uterus post-menarche, regardless of age. The LNG-IUS is effective for the treatment of dysmenorrhoea through menstrual suppression, resulting in amenorrhoea or a reduction in menstrual loss of at least 90 per cent by 12 months post-insertion. Insertion in a young woman who is not yet sexually 
active is performed under general anaesthetic or sedation. [37]

The LNG-IUS and Etonogestrel subdermal implant use is limited in adolescents. They are useful for heavy menorrhagia and dysmenorrhoea.

\section{Calcium Antagonists}

Nifedipine, a calcium channel blocker, inhibits myometrial contractility, thereby relieving primary dysmenorrhea. $68-70$ by blocking calcium entry into smooth muscle cell, intracellular free calcium is reduced, the muscle relaxes, contractions are reduced, vasodilatation is promoted, and ionic stimulation of prostanoids release is decreased. Adverse effects reported from the studies include transient facial flush, increased pulse rate, palpitations, and headache [38].

\section{Herbs}

Adolescents who drank rose tea (n70) perceived less menstrual pain and distress at 1,3 , and 6 months, compared with controls (n60). Other herbs, such as extracts of sweet fennel seeds (Foeniculum vulgare), are less potent than naproxen for relief of primary dysmenorrhea. A Cochrane analysis concluded that a small trial showed fish oil (mega-3 fatty acids) to be more effective than placebo. Krill oil significantly reduces the number of analgesics used for dysmenorrhea. Currently there is insufficient evidence to recommend the use of herbal and dietary therapies for dysmenorrhea [39].

\section{Nonpharmacologic Approaches}

Low fat vegetarian diet, dietary supplements with omega 3 fatty acids, increased physical activity heat therapy, acupuncture and Transcutaneous Electrical Nerve Stimulation (TENS) have been reported to improve dysmenorrhoea but the results are contradictory.

A three-pronged approach could be taken to better inform young women of dysmenorrhoea and associated support services. First, informative leaflets could be handed out to girls in schools by the school nurse so that adolescent females are familiar with the symptoms for dysmenorrhoea, which they can observe, and for which they can contact their GP if they require prescription pain medication. Second, health professionals could be more proactive in asking about this sensitive topic so that if young women have dysmenorrhoea they may be more likely to discuss their pain. Third, given the information-seeking behaviours reported in this sample, health professionals and schools could promote the use of reliable websites on which accurate information on dysmenorrhoea is published [40].
Risk factors for endometriosis in teenagers include obstructive Mullerian tract anomalies, family history, early menarche and early onset dysmenorrhea. Endometriosis in adolescents is suspected if the dysmenorrhoea is not responding to NSIADs, Dienogest and OCPs. Ultrasonography, Serum CA 125 level and MRI will help the diagnosis. Laparoscopy is diagnostic.

\section{Reproductive Tract Anomalies}

Any obstructive anomaly of the reproductive tract, whether hymenal, vaginal, or Müllerian, can cause secondary dysmenorrhea. The true incidence of reproductive tract anomalies is unknown, but they are estimated to occur in $0.1-3.8 \%$ of young women. Reproductive anomalies, both obstructive and nonobstructive, also are associated with endometriosis. Patients with urogenital sinus abnormalities and anorectal malformations are at increased risk of reproductive tract abnormalities and should be monitored for outflow tract obstructions as they progress through puberty and establish menses.

The goal of treatment in patients with obstructive reproductive tract anomalies is to establish a patent outflow tract to relieve symptoms and to decrease endometriosis and adhesions that can occur secondary to retrograde menses. Endometriosis associated with obstructive anomalies may improve after surgical correction and establishment of a patent outflow tract. However, persistent endometriosis after surgical correction of the obstructive anomaly has been reported [41]

\section{CONCLUSION}

The primary spasmodic dysmenorrhoea is painful menstrual period without any underlying pathological causes. Dysmenorrhoea among adolescent is not complained, not listened, underestimated and virtually untreated. Dysmenorrhoea can disturb their school attendance, performance, sports and other social activities. Dysmenorrhoea may have a negative impact on quality of life. The dysmenorrhoea can be treated with NSAIDs, progesterone COC and LNG-IUS. If they do not respond to these measures the possibility of secondary dysmenorrhoea due to endometriosis, PID, genital tract anomalies should be considered.

\section{REFERENCE}

1. Proctor, M., \& Farquhar, C. (2006). Diagnosis and management of dysmenorrhoea. BMJ. 332: 1134 1138.

2. Marjoribanks, J., Ayeleke, R. O., Farquhar, C., \& Proctor, M. (2015). Nonsteroidal antiinflammatory drugs for dysmenorrhoea. Cochrane Database Syst Rev. 7: CD001751.

3. Dawood, M. Y. (1981). Hormones, prostaglandin and dysmenorrhea. In: Dawood, M. Y. editor. 
Dysmenorrhea. Baltimore (MD): Williams and Wilkins; 20-52.

4. Sanfilippo, J., \& Erb, T. (2008). Evaluation and management of dysmenorrhea in adolescents. Clin Obstet Gynecol. 51(2):257-67.

5. Ortiz, M. I., Rangel-Flores, E., Carrillo-Alarcon, L. C., \& Veras-Godoy, H. A. (2009). Prevalence and impact of primary dysmenorrhea among Mexican high school students. Int J Gynaecol Obstet. 107:240-243.

6. Wong, L. P., \& Khoo, E. M. (2010). Dysmenorrhea in a multiethnic population of adolescent Asian girls. International Journal of Gynecology \& Obstetrics, 108(2), 139-142.

7. Mavrelos, D., \& Saridogan, E. (2013). Current therapeutic approaches to managing dysmenorrhoea. Prescriber, 24(12), 31-34.

8. Azagew, A. W., Kassie, D. G., \& Walle, T. A. (2020). Prevalence of primary dysmenorrhea, its intensity, impact and associated factors among female students' at Gondar town preparatory school, Northwest Ethiopia. BMC Women's Health, 20(1), 5.

9. Andersch, B., \& Milsom, I. (1982). An epidemiologic study of young women with dysmenorrhea. American journal of obstetrics and gynecology, 144(6), 655-660.

10. Widholm, O. (1979). Dysmenorrhea During Adolescence. Acta Obstetricia et Gynecologica Scandinavica; 58(Issue sup 87).

11. Ju, H., Jones, M., \& Mishra, G. (2014). The prevalence and risk factors of dysmenorrhea. Epidemiologic reviews, 36(1), 104113.

12. Burnett, M. A., Antao, V., Black, A., Feldman, K., Grenville, A., Lea, R., ... \& Robert, M. (2005). Prevalence of primary dysmenorrhea in Canada. Journal of Obstetrics and Gynaecology Canada, 27(8), 765-770.

13. Iacovides, S., Avidon, I., \& Baker, F. C. (2015). What we know about primary dysmenorrhea today: a critical review. Human reproduction update, 21(6), 762-778.

14. Harel, Z., Lilly, C., Riggs, S., Vaz, R., \& Drazen, J. (2000). Urinary leukotriene (LT) E4 in adolescents with dysmenorrhea: a pilot study. Journal of adolescent health, 27(3), 151154.

15. Willman, E. A., Collins, W. P., \& Clayton, S. G. (1976). Studies in the involvement of prostaglandins in uterine symptomatology and pathology. BJOG: An International Journal of Obstetrics \& Gynaecology, 83(5), 337-341.

16. Dawood, M. Y. (2006). Primary dysmenorrhea: advances in pathogenesis and management. Obstetrics \& Gynecology, 108(2), 428-441.

17. Balık, G., Üstüner, I., Kağıtcı, M., \& Şahin, F. K. (2014). Is there a relationship between mood disorders and dysmenorrhea?. Journal of pediatric and adolescent gynecology, 27(6), 371-374.

18. Azurah, A. G. N., Sanci, L., Moore, E., \& Grover, S. (2013). The quality of life of adolescents with menstrual problems. Journal of pediatric and adolescent gynecology, 26(2), 102-108.

19. Kitamura, M., Takeda, T., Koga, S., Nagase, S., \& Yaegashi, N. (2012). Relationship between premenstrual symptoms and dysmenorrhea in Japanese high school students. Archives of women's mental health, 15(2), 131-133.

20. Treloar, S. A., Bell, T. A., Nagle, C. M., Purdie, D. M., \& Green, A. C. (2010). Early menstrual characteristics associated with subsequent diagnosis of endometriosis. American journal of obstetrics and gynecology, 202(6), 534-e1.

21. Ylikorkala, O., \& Dawood, M. Y. (1978). New concepts in dysmenorrhea. American Journal of Obstetrics and Gynecology, 130(7), 833-847.

22. Osayande, A. S., \& Mehulic, S. (2014). Diagnosis and initial management of dysmenorrhea. American family physician, 89(5), 341-346.

23. Marjoribanks, J., Proctor, M., Farquhar, C., \& Derks, R. S. (2010). Non-steroidal antiinflammatory drugs for dysmenorrhoea. Cochrane Database Syst Rev. (1): CD 001751.

24. Sanfilippo, J., \& Erb, T. (2008). Evaluation and management of dysmenorrhea in adolescents. Clinical obstetrics and gynecology, 51(2), 257-267.

25. Marjoribanks, J., Ayeleke, R. O., Farquhar, C., \& Proctor, M. (2015). Nonsteroidal antiinflammatory drugs for dysmenorrhoea. Cochrane Database Syst Rev. 7: CD001751.

26. Zahradnik, H. P., Hanjalic-Beck, A., \& Groth, K. (2010). Nonsteroidal anti-inflammatory drugs and hormonal contraceptives for pain relief from dysmenorrhea: a review. Contraception, 81(3), 185-196.

27. Marjoribanks, J., Proctor, M., Farquhar, C., \& Derks, R. S. (2010). Nonsteroidal antiinflammatory drugs for dysmenorrhoea. Cochrane Database Syst Rev. (1):CD001751.

28. Subasinghe, A. K., Happo, L., Jayasinghe, Y. L., Garland, S. M., \& Wark, J. D. (2016). Prevalence and severity of dysmenorrhoea, and management options reported by young Australian women. Australian family physician, 45(11), 829834.

29. Ryan, S. A. (2017). The Treatment of Dysmenorrhea. Pediatr Clin North Am. 64(2):331-42.

30. Sasagawa, S., Shimizu, Y., Nagaoka, T., Tokado, H., Imada, K., \& Mizuguchi, K. (2008). Dienogest, a selective progestin, reduces plasma estradiol level through induction of apoptosis of granulosa cells in the ovarian dominant follicle without follicle-stimulating hormone suppression 
in monkeys. Journal of endocrinological investigation, 31(7), 636-641.

31. Osuga, Y., Hayashi, K., \& Kanda, S. (2020). Long-term use of dienogest for the treatment of primary and secondary dysmenorrhea. Journal of Obstetrics and Gynaecology Research, 46(4), 606617.

32. Milsom, I., \& Andersch, B. (1984). Effect of various oral contraceptive combinations on dysmenorrhea. Gynecologic and obstetric investigation, 17(6), 284-292.

33. Proctor, M. L., Roberts, H., \& Farquhar, C. M. (2001). Combined oral contraceptive pill (OCP) as treatment for primary dysmenorrhoea. The Cochrane Database of Systematic Reviews, (4), CD002120-CD002120.

34. Chan, W. Y., Dawood, M. Y., \& Fuchs, F. (1981). Prostaglandins in primary dysmenorrhea: comparison of prophylactic and nonprophylactic treatment with ibuprofen and use of oral contraceptives. The American journal of medicine, 70(3), 535-541.

35. Ekström, P., Åkerlund, M., Forsling, M., Kindahl, H., Laudanski, T., \& Mrugacz, G. (1992). Stimulation of vasopressin release in women with primary dysmenorrhoea and after oral contraceptive treatment-effect on uterine contractility. BJOG: An International Journal of Obstetrics \& Gynaecology, 99(8), 680-684.

36. Yiu, K. W., Chan, S. S., \& Chung, T. K. (2017). Mothers' attitude to the use of a combined oral contraceptive pill by their daughters for menstrual disorders or contraception. Hong Kong Med $J, 23(2), 150-7$.

37. Varma, R., Sinha, D., \& Gupta, J. K. (2006). Noncontraceptive uses of levonorgestrel-releasing hormone system (LNG-IUS) - A systematic enquiry and overview. Eur J Obstet Gynecol Reprod Biol. 125:9-28.

38. Sandahl, B., Ulmsten, U., \& Andersson, K. E. (1979). Trial of the calcium antagonist nifedipine in the treatment of primary dysmenorrhoea. Arch Gynecol. 227:147-51.

39. Yusoff, D. M. (2006). Primary Dysmenorrhea Advances in Pathogenesis and Management. Obstet Gynecol. 108:428-41.

40. Subasinghe, A. K., Happo, L., Jayasinghe, Y. L., Garland, S. M., Gorelik, A., \& Wark, J. D. (2016). Prevalence and severity of dysmenorrhoea, and management options reported by young Australian women. 45(11): 829-834.

41. Dietrich, J. E., Millar, D. M., \& Quint, E. H. Obstructive reproductive tract anomalies. J Pediatr Adolesc Gynecol. 2014; 27:396-402. 